\title{
Testing and modelling of the SVOM MXT narrow field lobster eye telescope optic
}

\author{
Charlotte Feldman ${ }^{\mathrm{a}}$, James Pearson ${ }^{\mathrm{a}}$, Richard Willingale ${ }^{\mathrm{a}}$, John Sykes ${ }^{\mathrm{a}}$, Paul Drummª, Paul \\ Houghton $^{\mathrm{a}}$, Chris Bicknell ${ }^{\mathrm{a}}$, Julian Osborne ${ }^{\mathrm{a}}$, Adrian Martindale ${ }^{\mathrm{a}}$, Paul O'Brien ${ }^{\mathrm{a}}$, Ray \\ Fairbend $^{\mathrm{b}}$, Emile Schyns ${ }^{\mathrm{b}}$, Sylvan Petit ${ }^{\mathrm{b}}$, Romain Roudot ${ }^{\mathrm{b}}$, Karine Mercier ${ }^{\mathrm{c}}$, Jean-Michel Le \\ Duigou $^{\mathrm{c}}$, and Diego Gotz \\ ${ }^{a}$ University of Leicester, University Road, Leicester, LE1 7RH, UK \\ ${ }^{\text {b} P h o t o n i s ~ F r a n c e ~ S . A . S ., ~ A v e n u e ~ R o g e r ~ R o n c i e r, ~} 19100$ Brive, B.P. 520, 19106 Brive Cedex, \\ France

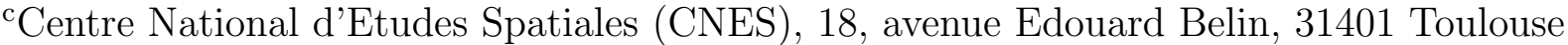 \\ Cedex 9 France \\ ${ }^{\mathrm{d}}$ CEA-Service d'Astrophysique, Orme des Merisiers, Bat. 709, 91191 Gif sur Yvette, France
}

\begin{abstract}
The Space-based multi-band astronomical Variable Objects Monitor (SVOM) is a French-Chinese space mission to be launched in 2021 with the goal of studying gamma-ray bursts, the most powerful stellar explosions in the Universe. The Microchannel X-ray Telescope (MXT) on-board SVOM, is an X-ray focusing telescope with a detector-limited field of view of $\sim 1$ square $^{\circ}$, working in the $0.2-10 \mathrm{keV}$ energy band. The MXT is a narrowfield-optimised lobster eye telescope, designed to promptly detect and accurately locate gamma-ray bursts. The breadboard MXT optic comprises of an array of square pore micro pore optics (MPOs) which are slumped to a spherical radius of $2 \mathrm{~m}$ giving a focal length of $1 \mathrm{~m}$ and an intrinsic field of view of $\sim 6^{\circ}$. We present details of the baseline design and results from the ongoing X-ray tests of the breadboard and structural thermal model MPOs performed at the University of Leicester and at Panter. In addition, we present details of modelling and analysis which reveals the factors that limit the angular resolution, characteristics of the point spread function and the efficiency and collecting area of the currently available MPOs.
\end{abstract}

Keywords: X-ray astronomy, X-ray telescopes, Microchannel Plate Optics

\section{INTRODUCTION}

SVOM is a satellite mission which is complemented by dedicated ground-based instrumentation and is designed specifically to study the physics of gamma-ray bursts (GRBs). The main objective of the SVOM mission is to better understand the GRBs by providing a spectrally and temporally well characterized multi-wavelength dataset for both prompt and afterglow emissions for more than 200 GRBs during the nominal 3 year mission lifetime. $^{1,2}$

GRBs are highly powerful and variable cosmological explosions which appear randomly across the sky. They are characterised by very short X-ray or $\gamma$-ray flashes lasting from a few seconds to a couple of hundred seconds, followed by a long-lasting afterglow observable from radio to X-rays.

SVOM includes an X-ray imaging telescope as one of the four payload instruments, the MXT, a narrow-fieldoptimised lobster eye soft X-ray telescope sensitive in the $0.2-10 \mathrm{keV}$ band and providing high sensitivity GRB afterglow observations with accurate high speed positioning.

The lobster eye geometry for X-ray imaging was first introduced by Angel (1979) ${ }^{3}$ and the use of MPOs in a lobster eye X-ray telescope was pursued by several authors. ${ }^{4-7}$ The square pore MPO devices used for the SVOM MXT are produced by Photonis France SAS. The geometry of the MPOs comprises a square packed array

Further author information: Charly Feldman:

E-mail: chf7@le.ac.uk, Telephone: +44 (0)116 2525084 

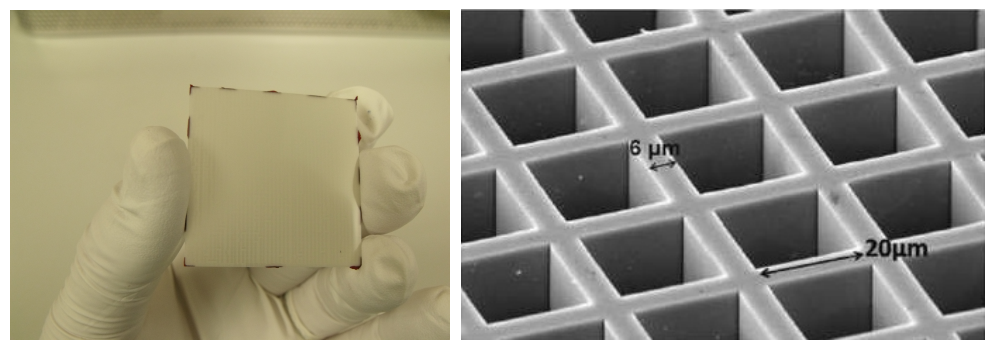

Figure 1. Left: A single, $40 \mathrm{~mm}$ by $40 \mathrm{~mm}$ square, bare glass micro pore optic. Right: An SEM image of a series of individual pores on a micro pore optic.

of microscopic pores with a square cross-section arranged over a spherical surface with a radius of curvature $\mathrm{R}$ $=2 \mathrm{~F}$, where $\mathrm{F}$ is the focal length of the optic. The aim is that all the pores should point towards a common centre of curvature with the reflections from the inside walls of the pores producing an image on a spherical focal surface with a radius of curvature F. For X-ray applications working in the photon energy range $0.2-10 \mathrm{keV}$ the optimum L/d ratio (length of pore $\mathrm{L}$ and pore width $\mathrm{d}$ ) is upwards of 50 . For wide-field lobster eye designs, the $\mathrm{L} / \mathrm{d}$ is constant across the whole optic aperture. In comparison, a narrow-field-optimised telescope has maximum area on-axis and the thickness of the MPOs is given by $\mathrm{L}=2.5 \mathrm{dF} / \mathrm{r}$ (where $\mathrm{r}$ is the radial position of the centre of the MPO from the centre of the optic). The MPOs for the MXT Proto-Flight Model (PFM) will have a pore width of $40 \mu \mathrm{m} ; 20 \mu \mathrm{m}$ pore width MPOs were also studied on the breadboard optic. To achieve an optimum $\mathrm{L} / \mathrm{d}$ of $>50$ with these pore sizes, the thickness of the MPOs are usually in the range of 1-2 $\mathrm{mm}$. An example of an MPO is shown in Figure 1.

The point spread function produced by a single MPO comprises of a focused spot, created by rays which undergo 2 grazing incidence reflections off adjacent sides of a pore, vertical and horizontal cross-arms, caused by rays which undergo 1 reflection, and a diffuse patch created by rays which pass straight-through the MPO.

The size of the field of view of a lobster eye optic depends only on the angular extent of the spherical optic and detector. Providing the optic is constructed with very small gaps between the tessellated MPOs for support, there is little vignetting and no change in the point spread function over the field of view. An example of a narrow field application is the SVOM MXT. ${ }^{2}$

\section{THE MXT BREADBOARD}

A breadboard of the SVOM MXT has been constructed. The frame of this optic accommodates an array of 21 square pore MPOs, in a $5 \times 5$ arrangement with the corners blanked out, each with an open aperture of $38 \mathrm{~mm}$ by $38 \mathrm{~mm}$ separated by $4 \mathrm{~mm}$ bars (left image of Figure 2). The MPOs are $40 \mathrm{~mm}$ x $40 \mathrm{~mm}$ allowing a $2 \mathrm{~mm}$ glue line for bonding. The radius of curvature of the frame is $2000 \mathrm{~mm}$ giving a focal length of $1000 \mathrm{~mm}$. A high precision aluminium frame has been produced with a machining accuracy of $\pm 10 \mu \mathrm{m}$ over the whole surface. The mass of the aluminium frame plus the 21 MPOs bonded on to it is $\sim 1 \mathrm{~kg}$.

For the purposes of the tests described below, 7 MPOs have been bonded to the frame. These included $2 \mathrm{x}$ $20 \mu \mathrm{m}$ pore width uncoated MPOs, $2 \times 40 \mu \mathrm{m}$ pore width uncoated MPOs, a $20 \mu \mathrm{m}$ Ir coated MPO and $2 \times 40$ $\mu \mathrm{m}$ pore width Ir coated MPOs. All MPOs have a thickness of $1.2 \mathrm{~mm}$. The layout of the MPOs on the frame are shown in the right image of Figure 2. A silicone adhesive is applied around the edges of the MPO to hold them to the frame. This adhesive provides a flexible interface in order to reduce the stress on the MPO due to the mechanical loads and thermal mismatch between the glass MPOs and the aluminium frame. Initially only small amounts of the adhesive were used to simply "tack" the MPOs to the frame.

The MPOs are formed by stacking a series of multifibres together, where the multifibres are an array of 25 x 25 pores for a pore width of $40 \mu \mathrm{m}(35 \mathrm{x} 35$ pores for a pore width of $20 \mu \mathrm{m})$. Both the breadboard and structural thermal model (STM) MPOs showed similar packing features, examples of which are shown in the microscope images in Figure 3. Due to the nature of the forces applied to adhere the individual multifibres to each other, the pores at the corners of the multifibre intersections, and a few surrounding pores, are not square in cross-section. 

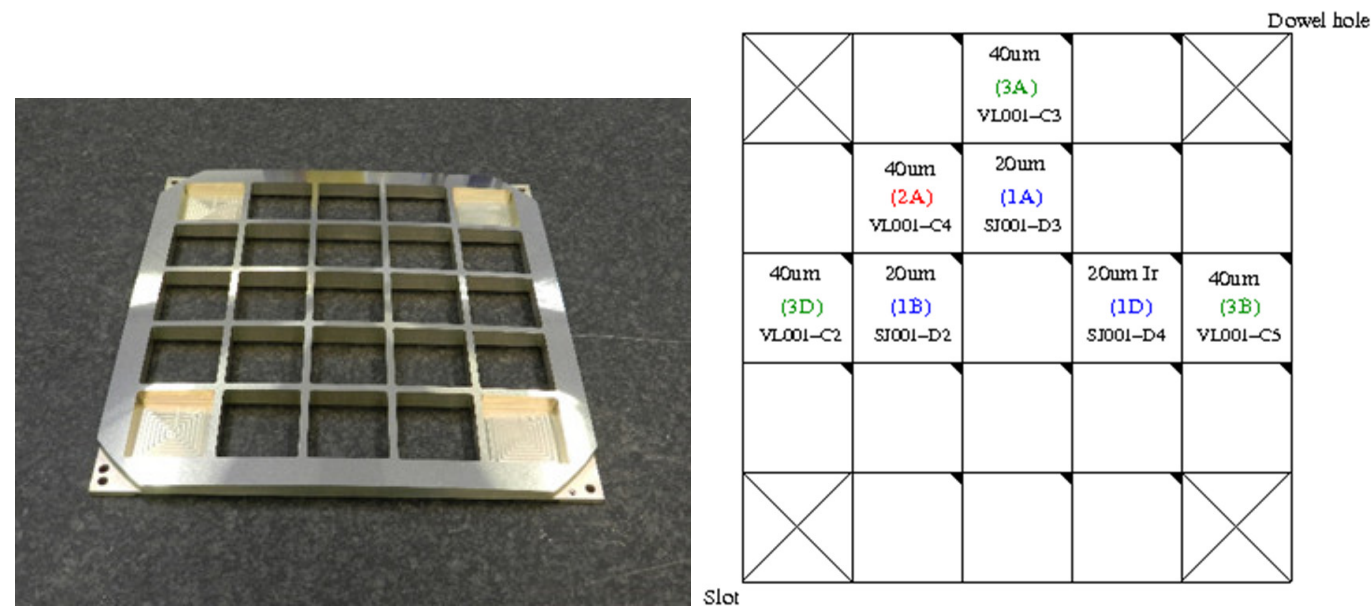

Figure 2. Left: The narrow field lobster eye breadboard frame Right: a schematic showing the positions of the bonded MPOs.

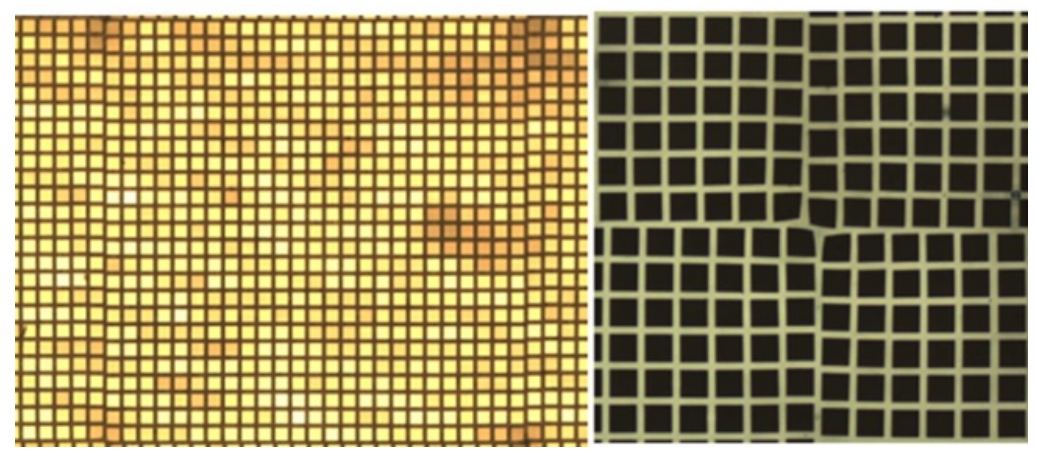

Figure 3. Left: a full $25 \times 2540 \mu \mathrm{m}$ pore multifibre on an STM MPO. Right: a microscope image centred on an MPO multifibre interface.

\subsection{MODELLING THE MPOS}

As described in Willingale et al., ${ }^{8}$ several features of the MPOs have been modelled and the models can produce an accurate representation of the X-ray images seen at both the University of Leicester and at Panter. By using this model and the physical properties of each individual MPO (measured radius of curvature, open fraction etc..), and with the MPO modelled off-axis to separate all components from each other (double reflection focus, cross-arms and straight-through patch), a prediction of the straight-through patch brightness fade ${ }^{8}$ and the number of counts expected in the double reflection focus can be achieved. By comparing these numbers to those in the real X-ray data image, a reflection ratio can be produced which indicates the performance of the reflecting surface and coating compared to the model of that MPO. The corner features seen at the multifibre intersections are included in the model as shear errors which radially propagate out from the multifibre corner.

A table of the modelled parameters and their contributions to the overall angular resolution is shown in Table 1. These combines to a FWHM of 6.5', slightly better than the average performance measured on the breadboard MPOs (see sections 2.2 and 2.3). We are currently unable to split figure errors and shear errors.

An X-ray image of an MPO is shown in the left hand image of Figure 4 alongside a modelled image of the same MPO, in the centre. This is a $1.7 \mathrm{~mm}$ thick, Ir coated STM MPO with a pore width of $40 \mu \mathrm{m}$. The surface brightness fade (as described in Willingale et al. ${ }^{8}$ ), both simulated (solid line) and X-ray (points), on the right hand side of Figure 4.

\subsection{BREADBOARD TESTS AT LEICESTER}

The University of Leicester has a $27 \mathrm{~m}$ long X-ray beam line (the TTF) capable of producing X-rays in the range of 0.5-100 keV and ending in an experiment chamber with mounting for individual MPOs or the full MXT optic 


\begin{tabular}{lr}
\hline geometric pore size $\mathrm{d}=20 \mu \mathrm{mm}$ & 0.07 \\
spherical aberration pore L/d $=50$ & 0.15 \\
slumping - intrinsic radial tilt errors & 0.17 \\
slumping - intrinsic shear errors & 0.60 \\
surface roughness 11 Årms, $\omega_{b}=10 \mathrm{~mm}^{-1}$ & 2.46 \\
figure errors at centre of multifibres & 2.48 \\
shear errors at centre of multifibres & - \\
multifibre tilt errors along primary axes & 3.54 \\
figure errors at edge of multifibres & 9.92 \\
shear errors at edge of multifibres & - \\
global shear errors & - \\
global tilt errors & - \\
\hline
\end{tabular}

Table 1. Contributions to the angular resolution FWHM that combine to give the measured performance of 6.5'.
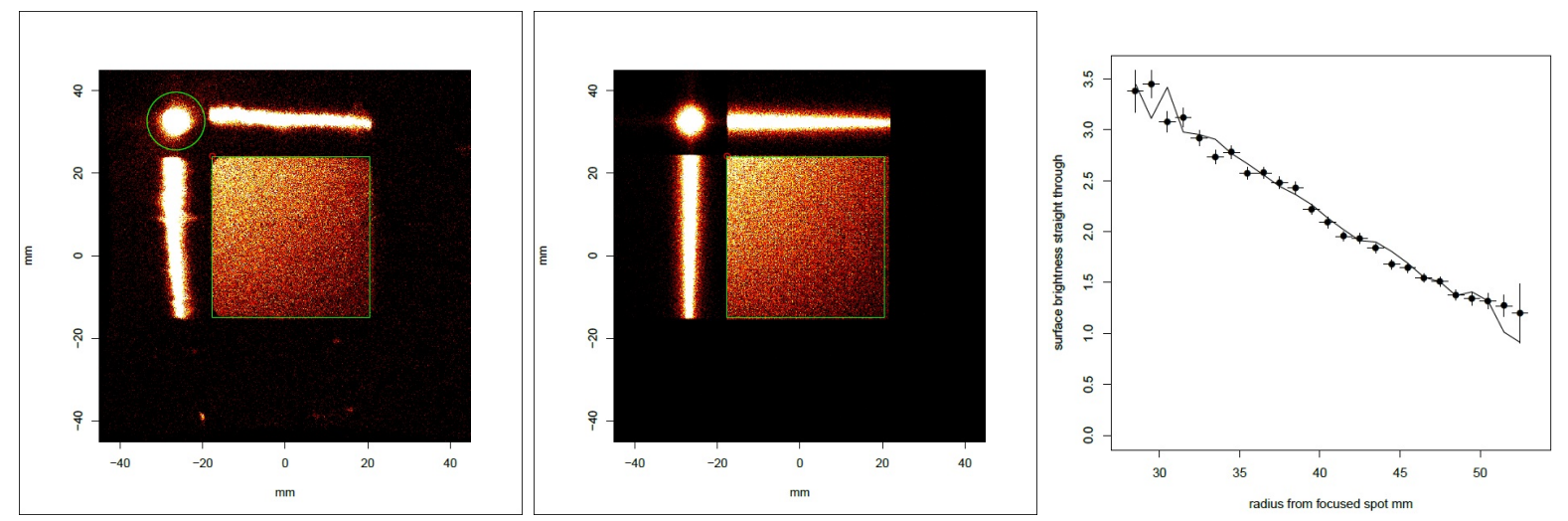

Figure 4. Left: An X-ray image of a $1.7 \mathrm{~mm}$ thick, Ir coated STM MPO with a pore width of $40 \mu \mathrm{m}$. Centre: A simulation using the model described in Section 2.1 of the same STM MPO. Right: The simulated and X-ray surface brightness fade of this MPO.

frame and an imaging MCP detector. The detector chamber opens in to a ISO class 5 clean room to ensure a continual clean environment for the MPOs and MXT optic. A $1.49 \mathrm{keV} \mathrm{Al-K} \mathrm{line} \mathrm{can} \mathrm{be} \mathrm{produced} \mathrm{by} \mathrm{the} \mathrm{soft}$ $\mathrm{X}$-ray source and all the X-ray tests have been performed at this energy.

Each MPO on the frame was studied on-axis and off-axis and its X-ray focal length was calculated. Combinations of MPOs were also studied to look at the common focus and to confirm the alignment obtained during bonding of the MPOs and the accuracy of the frame radius. The optic frame focus and the foci of the MPOs should be in the same position as their slump radii should match. However, if the MPO slump radius does not match that of the optic frame then the common focus should be at the focus position of the optic frame and not the individual MPOs, as the MPOs are not forced to conform to the optic frame radius. In this case the point spread function (PSF) of the combined focus is increased and blurred. With a source at an infinite distance, for the breadboard and qualification model (QM - using the STM MPOs) optics, the focus will be at $1000 \mathrm{~mm}$. But with a finite source distance within the beam line at the University of Leicester or within the Panter test facility, the focus is closer to the MPOs by $36 \mathrm{~mm}$ and $6 \mathrm{~mm}$ respectively. The 2A and 3B MPOs on the breadboard were used to test the alignment and the accuracy of the frame radius of curvature, as they are both Ir coated and are at widely separated positions on the frame. The left hand image of Figure 5 shows the two MPOs not combining to a central focus but highlights the different components from both MPOs. With a small adjustment of the focal length of the optic, it was possible to bring the foci in to line as shown in the right hand image of Figure 5, and was repeated at the Panter test facility. The two MPOs came to a common focus at the expected optic to detector distances for the respective facilities.

Using the models described in Section 2.1 and the X-ray images obtained within the TTF, the average breadboard MPO FWHM was found to be $\sim 7$ ' with a reflection ratio of $\sim 62 \%$. The reflection ratios obtained 


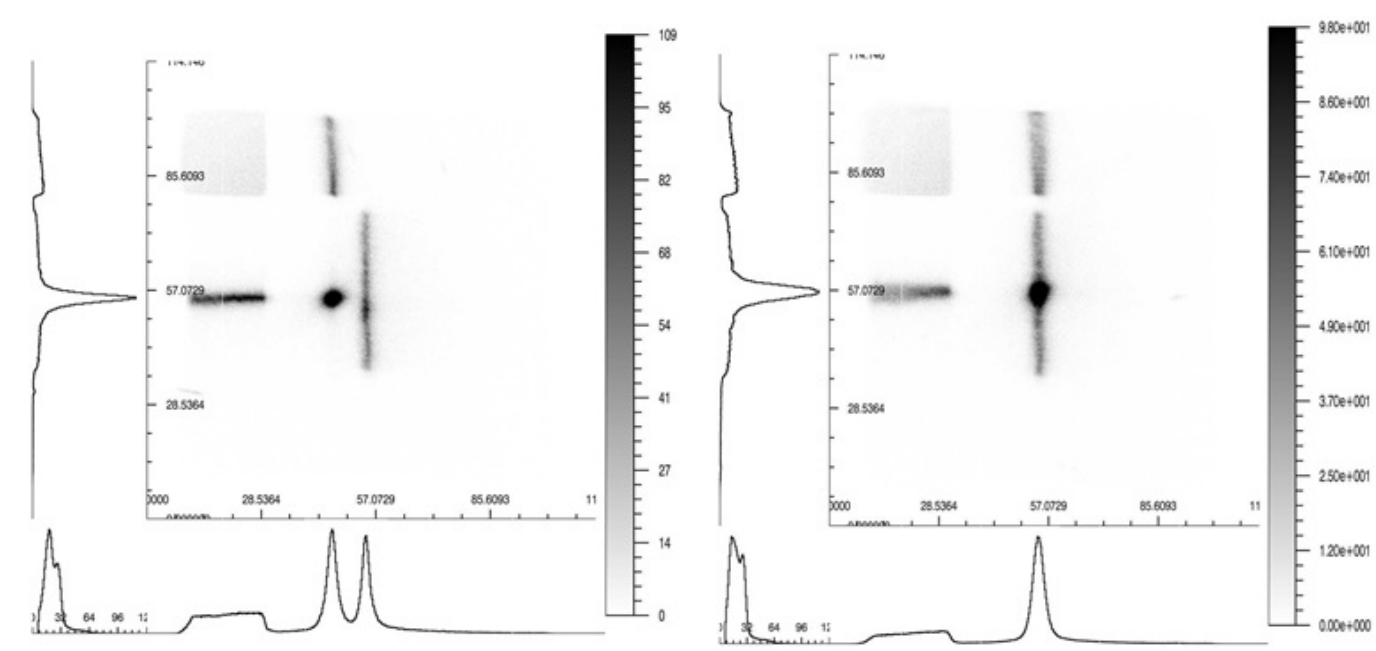

Figure 5. Left: MPO 2A and MPO 3B focussing but at the incorrect optic to detector distance, at the University of Leicester. Right: MPO 2A and MPO 3B focussing together at the correct optic to detector distance.

\begin{tabular}{cc|c|c|c} 
& & Double reflection ratio & FWHM (arcmin) & HEW $(\operatorname{arcmin})$ \\
\hline TTF & Corner 1 & 0.51 & 7.4 & 10.6 \\
TTF & Corner 2 & 0.45 & 7.0 & 11.1 \\
\hline Panter & Corner 1 & 0.47 & 7.8 & 10.9 \\
Panter & Corner 2 & 0.49 & 7.2 & 11.1 \\
\hline
\end{tabular}

Table 2. Results from two opposite corners of MPO 3B at both the University of Leicester and Panter facilities

varied greatly between the Ir coated MPOs (average of $64 \%$ ) and the bare glass MPOs (average of 25\%). However, the FWHMs were reasonably consistent across the 7 MPOs on the breadboard, with six ranging from 6.5' to 7.7' and one with a FWHM of 9.8 '.

Figure 6 shows X-ray images of two opposite corners from the MPO 3B obtained at both the University of Leicester and at Panter. Two opposite corners of the Ir coated MPO were studied as this samples all four walls of each pore. These images were taken at 45' off-axis in both axes to position the double reflection spot away from the straight-through patch. All the images were taken at Al-K (1.49 keV), but the CCD detector at Panter is much smaller than the MCP detector at the University of Leicester so a smaller area is visible. The images are remarkably similar, showing the repeatability of results between the two facilities.

Using the analysis software developed using the models of the MPOs described in Section 2.1, the X-ray images from each of the corners from each of the facilities were analysed to calculate the reflection ratio; these are shown in Table 2. The difference in the double reflection ratio between the two sides of the TTF results are due to a variation in the efficiency across the detector caused by the bias angle in the horizontal axes of the MCP detector.

The temperature sensitivity of the optic has also been examined within the TTF. Two MPOs on the MXT breadboard were tested, 2A and 3B. Each MPO was tested in a fully bonded configuration using a continuous glue line around the perimeter of the MPO.

The breadboard frame was cooled by circulating a temperature controlled water/glycol mix through copper pipes bonded into an aluminium cooling plate (Figure 7). The cooling plate was bolted onto the mounting used to secure the breadboard frame within the TTF. The temperature of the frame was measured using a platinum resistance thermometer (PRT) bonded onto the edge of the frame using Stycast 2850. Two identical PRTs were fitted to the tank flange in the clean room and the beampipe close to the tank. The temperature of the fluid was controlled using an ATC $\mathrm{K} 1$ chiller which has an operational range of $+4^{\circ} \mathrm{C}$ to $+35^{\circ} \mathrm{C}$. Additional high and low temperature data points were obtained on MPO $2 \mathrm{~A}$ using a Lauda Alpha RA8 chiller. Measurements of 

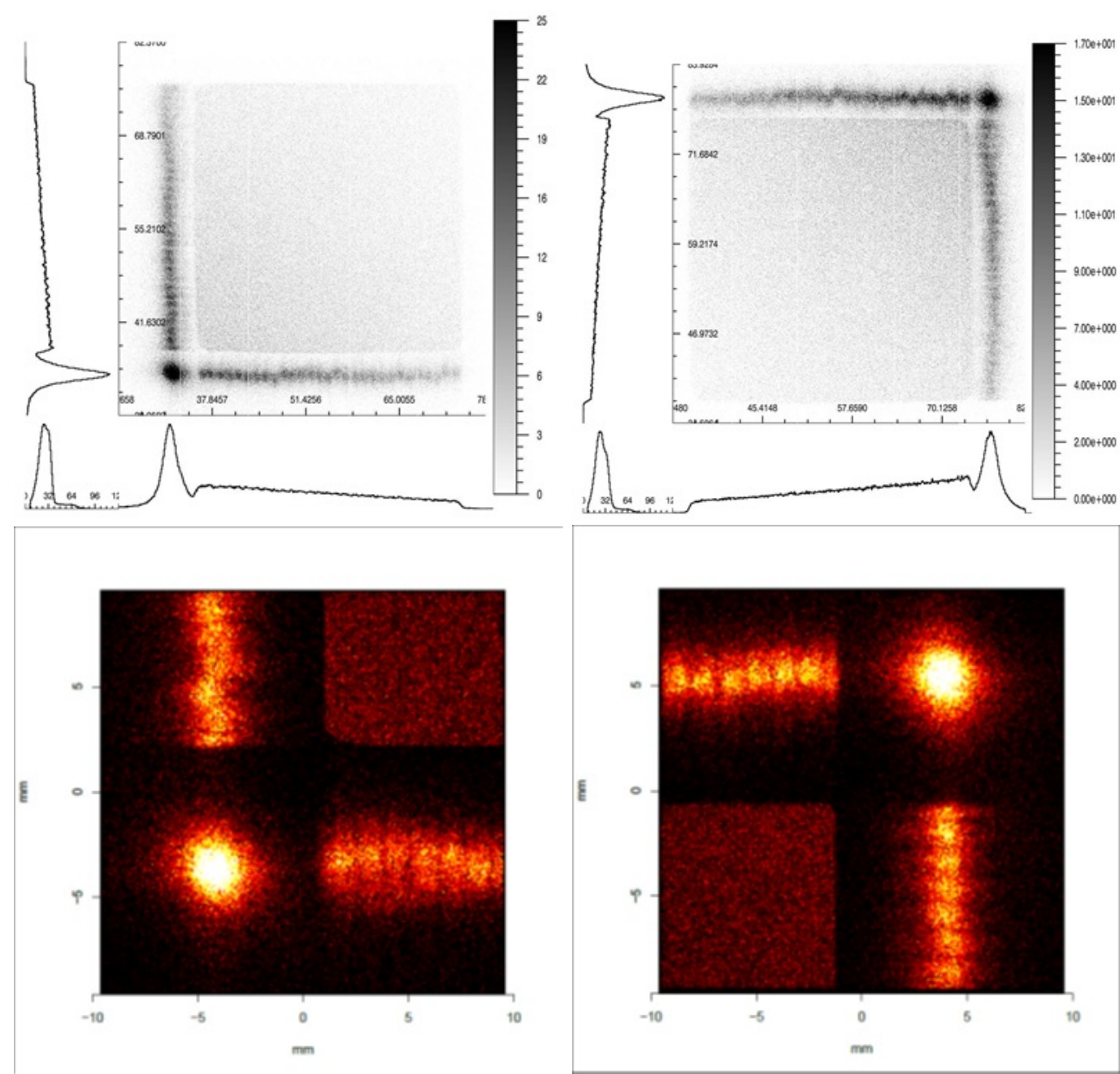

Figure 6. Top left: MPO 3B corner $14^{\prime}$ ' off-axis in both directions at University of Leicester. Top right: Corner 2 at University of Leicester. Bottom left and bottom right are repeats of the images in the top left and the top right taken at Panter. All taken at Al-K.

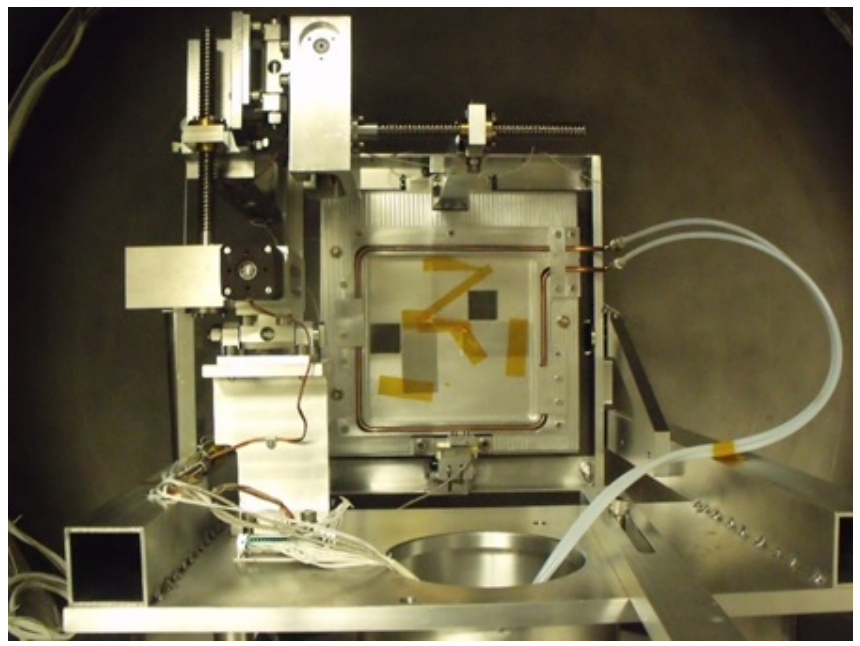

Figure 7. The temperature test set-up within the TTF at the University of Leicester. 
Breadboard MPO 2A full bond

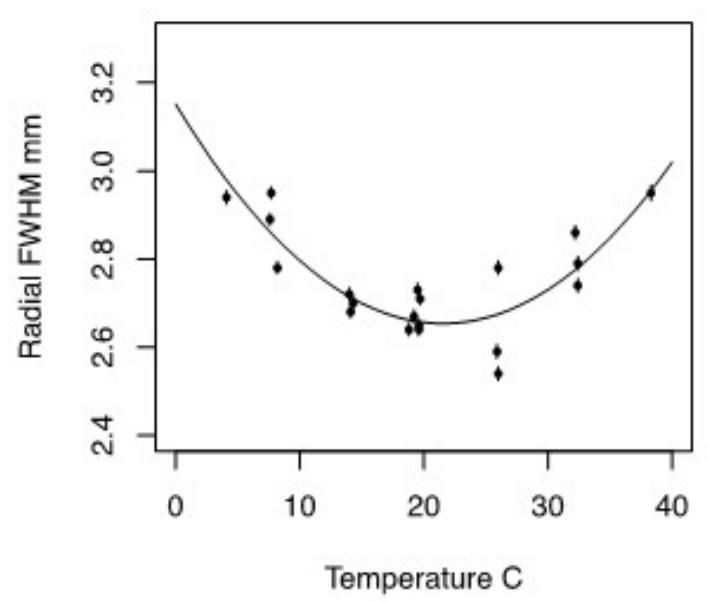

Breadboard MPO 2A full bond

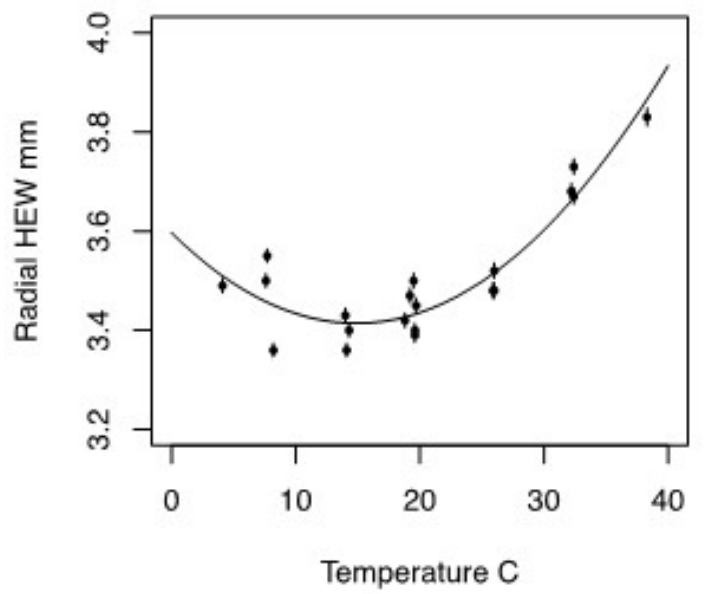

Breadboard MPO 2A full bond

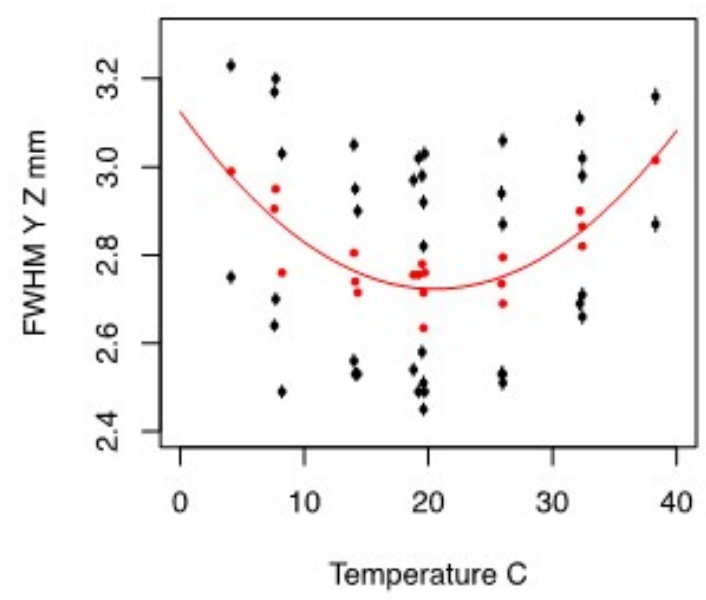

Breadboard MPO 2A full bond

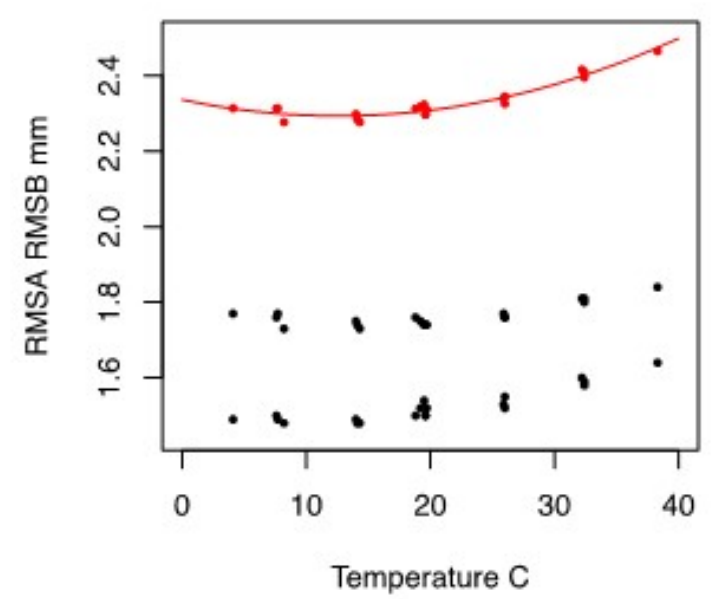

Figure 8. Spot size variation with temperature of MPO 2A fully bonded.

the MPO's FWHM was made at various temperatures in the range of $0^{\circ} \mathrm{C}$ and $40^{\circ} \mathrm{C}$. These temperatures were allowed to stabilise over several hours so they were constant whilst each X-ray image was taken.

As the thermal conductivity of aluminium is high and the cooling plate was surrounded by the copper pipes, it is not expected that there would be any significant temperature gradients across the breadboard frame. The temperature of the MPOs was not measured during the experiment. Given the duration of the tests it is likely that the MPOs reached a temperature close to that of the frame, although the conductive coupling between the frame and the MPOs is not high.

The results obtained from MPO 2A fully bonded to the breadboard frame are shown in Figure 8 . The left hand image shows the radial FWHM whilst the right hand image shows the FWHM in Y and Z in black and the average ((FWHMY + FWHMZ)/2) plotted in red. The curves plotted are least squares quadratic fits to the data points.

Under the test conditions a fully bonded MPO of thickness $1.2 \mathrm{~mm}$ has a double reflection FWHM temperature 
sensitivity of $0.27^{\prime} /{ }^{\circ} \mathrm{C}$. If the FWHM is $7^{\prime}$ (average of the breadboard MPOs), this sensitivity would give a FWHM of $7.5^{\prime}$ at $\pm 10^{\circ} \mathrm{C}$ away from the temperature at which the optics were bonded to the aluminium frame. Therefore the MXT optic will be maintained at a temperature of $20^{\circ} \mathrm{C} \pm 10^{\circ} \mathrm{C}$ during orbit to be as close to assembly temperature as possible. The thermal modelling of the MPOs and optic frame suggests that there will be a variation in temperature across the MPOs as the glass has a poor thermal conductivity. The temperature at the centre of the optic is determined more by radiation as opposed to conduction. For this reason the temperature control of the optics on the aluminium frame will be a combination of heating the top of the telescope tube and a heater on the rear of the aluminium optics frame.

\subsection{BREADBOARD TESTS AT PANTER}

A one-week testing programme was undertaken at the Panter test facility at MPE, Neuried, Germany. Images were taken using both the Tropic CCD and the PSPC detector. Due to the small size of the Tropic CCD, in order to produce comparable images to those obtained in the TTF, several Tropic images were stitched together to form a complete image. Tests were carried out to confirm the co-alignment of the MPOs, the change in efficiency due to a change in the angle of reflection, the change in efficiency due to a change in energy and to examine the differences between the Ir coated MPOs and the bare glass MPOs. A subset of the results from the tests carried out at various energies are presented here.

The energies studied were the lines of C-K (0.28 keV), Al-K (1.49 keV), Ag-L (2.98 keV), Ti-K $\alpha$ (4.51 keV) and $\mathrm{Ti}-\mathrm{K} \beta(4.93 \mathrm{keV})$, Fe-K $(6.40 \mathrm{keV})$ and $\mathrm{Cu}-\mathrm{K}(8.05 \mathrm{keV})$. The MPO used for these tests was 3B (in the right image of Figure 2), an Ir coated, $40 \mu \mathrm{m}$ width pore, $1.2 \mathrm{~mm}$ thick MPO. As with the images shown in the top left and top right of Figure 6 obtained within the TTF, two opposite corners of the MPO were studied at 45' off-axis in both axes at each energy. As shown in Figure 9, there is a large variation in the number of counts obtained at each of the energies even though the images were taken for the same amount of time. The variation in the number of counts limited the accuracy of the results. Using these images, and information about the source flux rate, it was possible to normalise the data and calculate the relative reflection efficiency of the MPO at each energy. A comparative model of an MPO with similar form parameters was created for comparison with experimental data (Figure 10). The difference between the model and X-ray data is due to the Ir coating having $\sim 50 \%$ reflection ratio as shown in Table 2 .

\section{STM MPO TESTING AT LEICESTER}

The QM model of the MXT optic consists of a tessellation of the 21 STM MPOs, which have 4 thicknesses of $1.05 \mathrm{~mm}, 1.2 \mathrm{~mm}, 1.7 \mathrm{~mm}$ and $2.4 \mathrm{~mm}$ and a poor width of $40 \mu \mathrm{m}$. The design of the QM is shown in Figure 11. These MPOs have been slumped to a spherical radius of $2000 \mathrm{~mm}$ to have a focal length of $1000 \mathrm{~mm}$. All 21 STM MPOs have been cut from the same block of material in order to maintain a consistent residual bias angle between MPOs thus avoiding the necessity of individual MPO alignment. Each MPO has been individually tested within the TTF at $1.49 \mathrm{keV}$, looking at the X-ray focal length, angular response and FWHM. Photonis France SAS have provided data on the slump radius, pitch of pores and average pore width which has enabled accurate models for each individual MPO to be created. Using these models and the X-ray data obtained, the reflection ratio for each MPO has been calculated.

Examples of X-ray images obtained at the University of Leicester are shown in Figure 12. In comparison to the results seen with the breadboard MPOs (Figure 6), there appears to be little to no modulation in the cross-arms, which do broaden towards the edges furthest from the focus spot. Curvature and non-orthogonality of the cross-arms. Even with these changes in features, the reflection ratio is high at $\sim 90 \%-100 \%$, and this value and the PSF width of the MPOs is consistent across the full 21 STM MPO batch. A significant variation in the radius of curvature across the 21 MPOs was noted, most evidently in the 1.05 mm MPOs.

\section{SUMMARY AND CONCLUSION}

The MXT is one of four payload instruments on the SVOM satellite. It is a narrow-field-optimised lobster eye Xray telescope to provide accurate positioning and imaging of GRBs in the energy range of $0.2-10 \mathrm{keV}$. The MXT optic has low vignetting and has a low mass (aluminium frame plus MPOs), with the complete optic assembly 

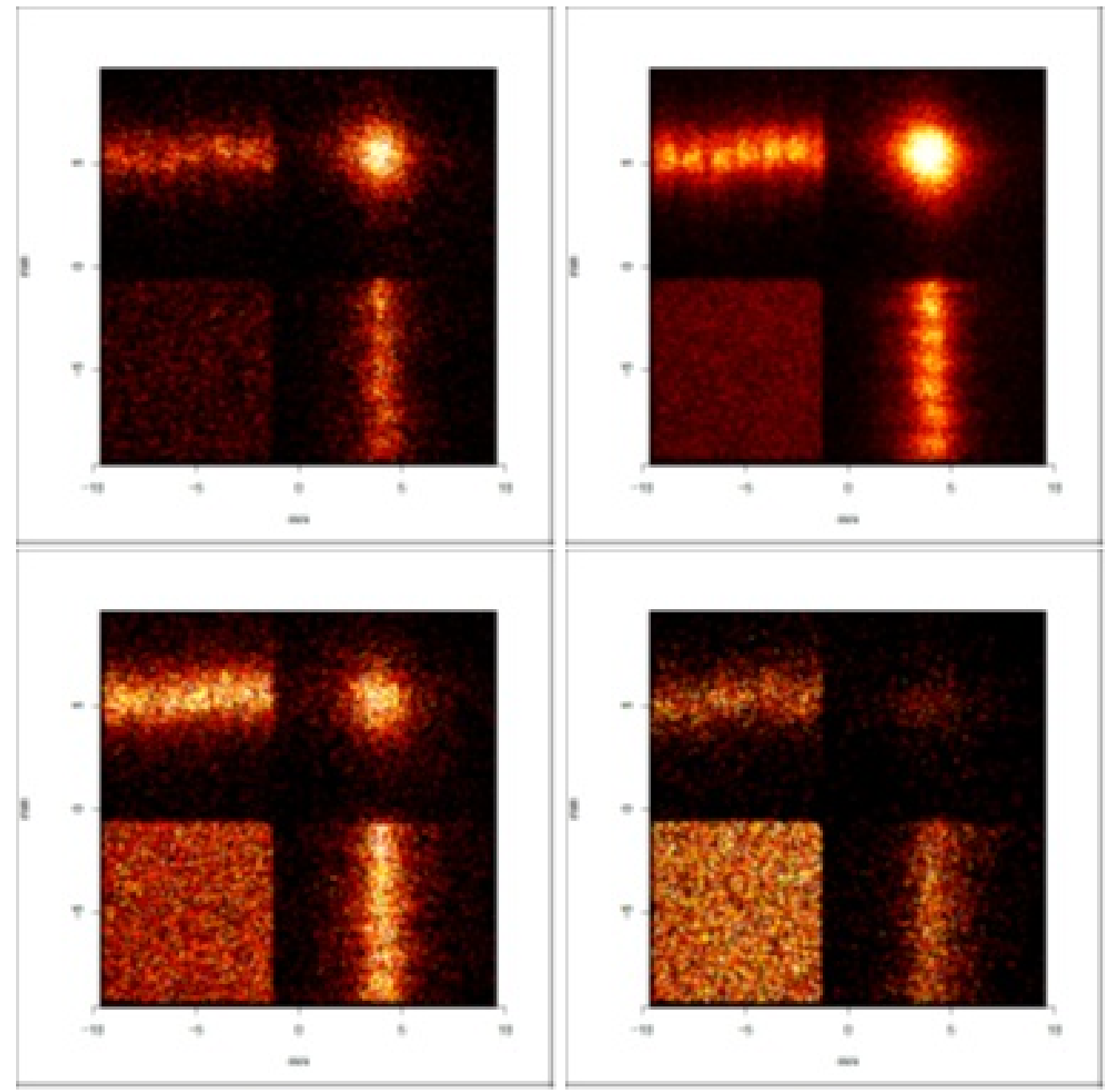

Figure 9. Images obtained using the Tropic CCD at Panter of corner 2 of MPO 3B (Figure 2) at energies of C-K, Al-K, $\mathrm{Ag}-\mathrm{L}$ and $\mathrm{Cu}-\mathrm{K}$ (from left to right, top to bottom). 


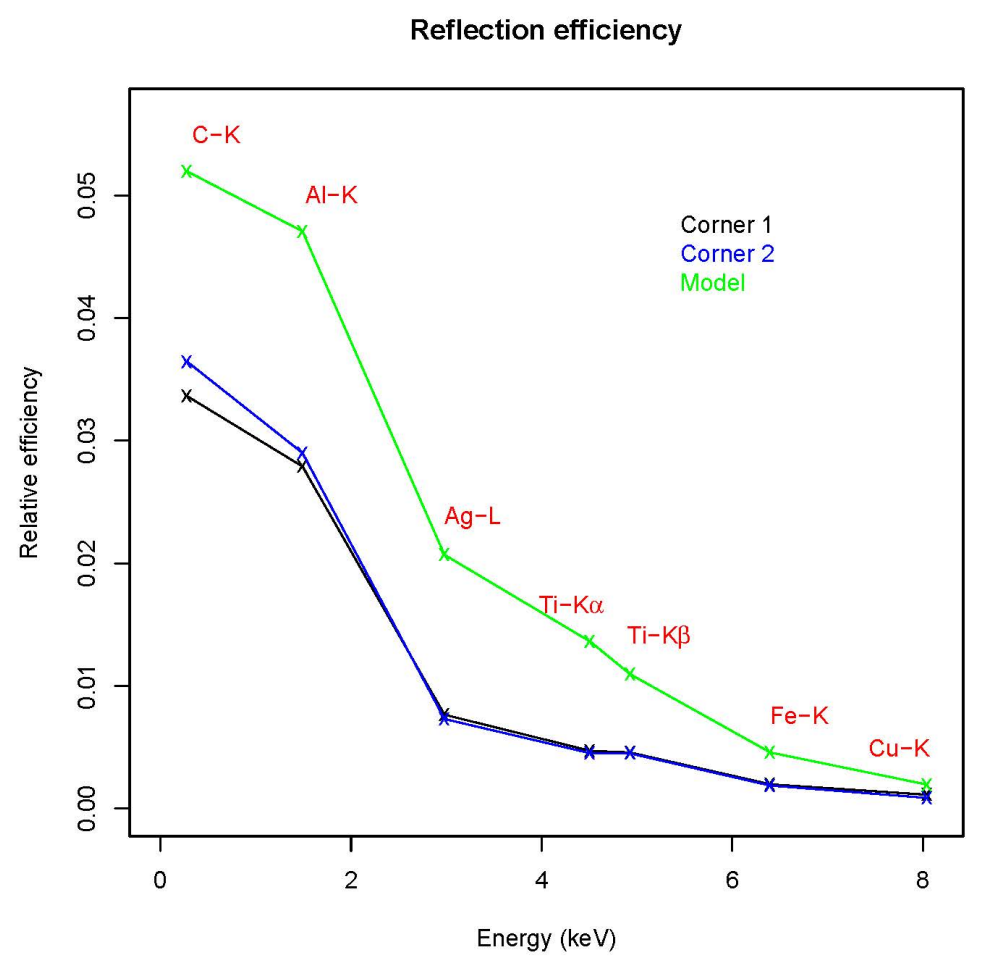

Figure 10. The relative efficiencies of two corners of the MPO 3B, at each energy tested at the Panter test facility, and a comparative model of a similar MPO.

\begin{tabular}{|c|c|c|c|c|}
\hline & $\begin{array}{c}4 \mathrm{~A} \\
1.06 \quad 27\end{array}$ & $\begin{array}{c}3 \mathrm{~A} \\
1.1930\end{array}$ & $\begin{array}{c}4 \mathrm{~B} \\
1.06 \quad 27\end{array}$ & \\
\hline $\begin{array}{r}4 \mathrm{H} \\
1.06 \quad 27\end{array}$ & $\begin{array}{r}2 \mathrm{~A} \\
1.68 \quad 42\end{array}$ & $\begin{array}{r}1 \mathrm{~A} \\
2.38 \quad 60\end{array}$ & $\begin{array}{c}2 \mathrm{~B} \\
1.68 \quad 42\end{array}$ & $\begin{array}{c}4 \mathrm{C} \\
1.06 \quad 27\end{array}$ \\
\hline $\begin{array}{r}3 \mathrm{D} \\
1.19 \quad 30\end{array}$ & $\begin{array}{r}1 \mathrm{~B} \\
2.38 \quad 60\end{array}$ & $\begin{array}{c}1 \mathrm{C} \\
{ }_{2.38} \quad 60\end{array}$ & $\begin{array}{r}1 \mathrm{D} \\
2.38 \quad 60\end{array}$ & $\begin{array}{c}3 \mathrm{~B} \\
1.19 \quad 30\end{array}$ \\
\hline \multirow[t]{2}{*}{$\begin{array}{c}4 \mathrm{G} \\
1.06 \quad 27\end{array}$} & $\begin{array}{c}2 \mathrm{D} \\
1.68 \quad 42\end{array}$ & $\begin{array}{c}1 \mathrm{E} \\
2.38 \quad 60\end{array}$ & $\begin{array}{c}2 \mathrm{C} \\
1.68 \quad 42\end{array}$ & $\begin{array}{c}4 \mathrm{D} \\
1.06 \quad 27\end{array}$ \\
\hline & $\begin{array}{c}4 \mathrm{~F} \\
1.06 \quad 27\end{array}$ & $\begin{array}{c}3 C \\
1.1930\end{array}$ & $\begin{array}{c}4 \mathrm{E} \\
1.06 \\
27\end{array}$ & \\
\hline
\end{tabular}

Figure 11. The design of the QM MXT optic with a focal length of $1000 \mathrm{~mm}$. Each MPO is shown with it's label, thickness in $\mathrm{mm}$ (lower left number) and the $\mathrm{L} / \mathrm{d}$ ratio (lower right number). 

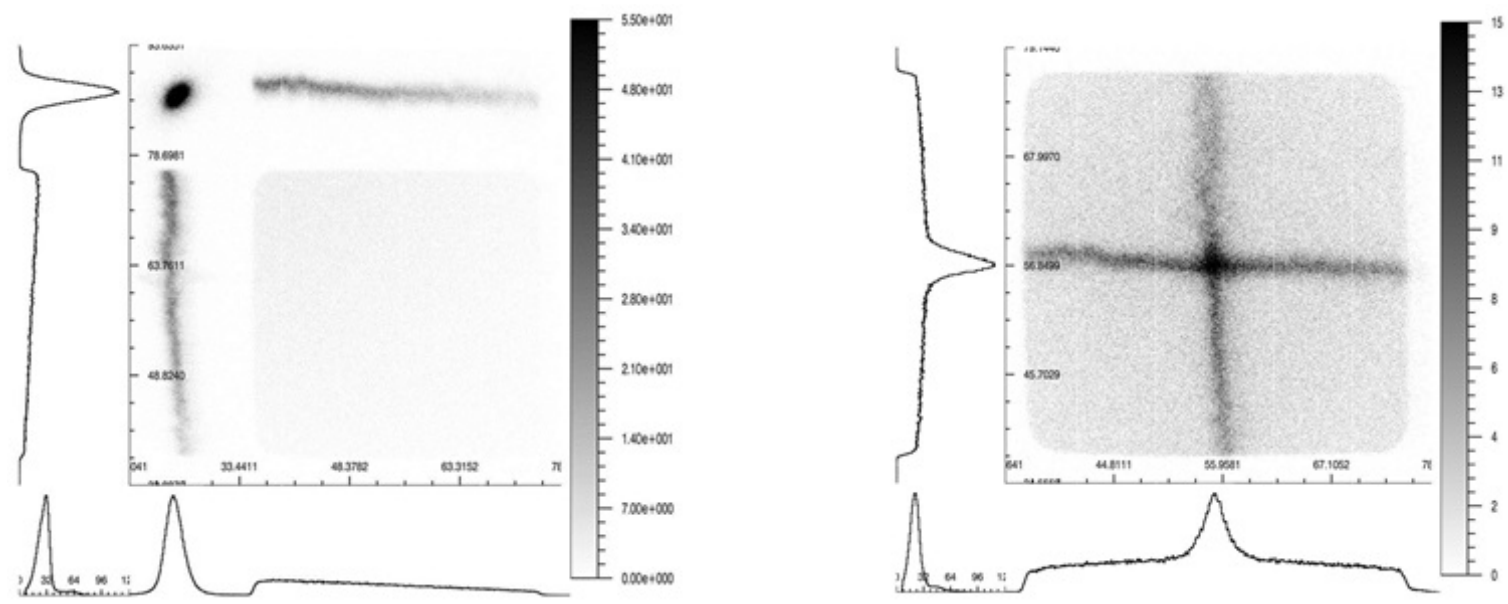

Figure 12. Examples of X-ray data obtained within the TTF at the University of Leicester of a $1.2 \mathrm{~mm}$ thick, Ir coated STM MPO at $1.49 \mathrm{keV}$.

weighing $\sim 1 \mathrm{~kg}$. A breadboard of the SVOM MXT has been constructed and tested at both the University of Leicester and at Panter. The X-ray data has shown that the MPOs have been successfully aligned on an accurate spherical surface and the data was confirmed at Panter. Temperature stability measurements were completed with the MPO fully bonded to the aluminium frame, the results of which have shown that the MXT optic should be kept within a temperature range of $20^{\circ} \mathrm{C} \pm 10^{\circ} \mathrm{C}$ in flight. All $21 \mathrm{STM}$ MPOs have been individually tested and although further analysis of all data is required. The STM MPOs reflection ratio suggests that the theoretical effective area $\left(\mathrm{cm}^{2}\right.$ at $\left.1 \mathrm{keV}\right)$ will be achieved.

The QM build is about to start now all STM MPOs have been individually tested, the completed optic will be tested at Panter in the third quarter of 2018. The PFM MPOs are currently being made and are expected to be delivered for testing at the University of Leicester in the first quarter of 2018.

\section{ACKNOWLEDGEMENTS}

The authors wish to thank the rest of the SVOM team and the entire team at Panter for their support and immense help they provided during the X-ray tests.

This research used the SPECTRE High Performance Computing Facility at the University of Leicester.

\section{REFERENCES}

[1] K. Mercier, F. Gonzalez, M. Jouret-Perl, J. Atteia, P. Mandrou, R. Pons, S. Basa, B. Cordier, D. Gotz, F. Pinsard, S. Schanne, C. Lachaud, J. Wei, and S. Zhang, "The french payload on-board the svom frenchchinese mission," Proc. of SPIE 9144, 2014.

[2] D. Gotz, C. Adami, S. S. Basa, V. Beckmann, V. Burwitz, R. Chipaux, B. Cordier, P. Evans, O. Godet, R. Goosmann, N. Meidinger, A. Meuris, C. Motch, K. Nandra, P. OBrien, J. Osborne, E. Perinati, A. Rau, R. Willingale, K. Mercier, and F. Gonzalez, "Microchannel x-ray telescope on board the svom satellite," ArXiv e-prints , 2015.

[3] J. Angel, "Lobster eyes as x-ray telescopes," Ap. J. 233, pp. 364-373, 1979.

[4] H. Chapman, K. Nugent, and S. Wilkins, "X-ray focusing using cylindrical-channel capillary arrays, i. theory," Applied Optics 32, p. 6316, 1993.

[5] S. Wilkins, A. Stevenson, K. Nugent, H. Chapman, and S. Steenstrup, "On the concentration, focusing, and collimation of x-rays and neutrons using microchannel plates and configurations of holes," Review of scientific instruments 60, pp. 1026-1036, 1989.

[6] G. W. Fraser, J. E. Lees, J. F. Pearson, M. R. Sims, and K. Roxburgh, "X-ray focusing using microchannel plates," Proc. of SPIE 1546, 1992. 
[7] P. Kaaret, P. Geissbuhler, A. Chen, and E. Glavinas, "X-ray focusing using microchannel plates," Appl.Opt. 31, 1992.

[8] R. Willingale, J. F. Pearson, A. Martindale, C. H. Feldman, R. Fairbend, E. Schyns, S. Petit, J. P. Osborne, and P. T. O'Brien, "Aberrations in square pore micro-channel optics used for x-ray lobster eye telescopes," Proc. of SPIE 9905, 2016. 